\title{
A Novel RFID-Based Wireless Health Monitor by Measurement of Acupuncture Bio-Potentials with Array Probes on Tags and Different Wearable Fixtures
}

\author{
Jium-Ming Lin ${ }^{1}$ and Cheng-Hung Lin $^{2}$ \\ ${ }^{1}$ Department of Communication Engineering, Chung-Hua University, Hsin-Chu 30012, Taiwan \\ ${ }^{2}$ Ph.D. Program in Engineering Science, College of Engineering, Chung-Hua University, Hsin-Chu 30012, Taiwan \\ \{jmlin \& b09306014\}@chu.edu.tw
}

\begin{abstract}
In this paper a bio-potential monitor system is presented, which relates more particularly to a bio-potential measurement system having wireless communication capability. The bio-potential measurement device includes a flexible substrate (e.g. an active RFID tag) configured to be fastened around a body portion, two probe sets attached to the flexible substrate, a measurement device, and a wireless device. One probe set includes micro-array probes with tip-portions to pierce the skin adjacent to an acupuncture point. Another probe set is used to contact the reference grounded skin. The measurement device is disposed on the flexible substrate and electrically coupled to the two probe sets to detect the biopotentials at the acupuncture points. The measurement device can amplify and measure the bio-potentials across the acupuncture points and the ground. The wireless device, coupled to the measurement device, is configured to transmit the acupuncture code and biopotential information to a remote monitor station for health analysis and care. Several wearable fixtures on the body are also proposed.

Index Terms - Active RFID tag. Flexible substrate. Biopotential. Acupuncture point. Array probes. Wearable fixtures.
\end{abstract}

\section{Introduction}

In 1950s, Dr. Reinhard Voll studied acupuncture points and discovered nearly 2000 acupuncture points on the skin of a human body [1-2], and those acupuncture points are distributed along the paths called meridians. Fig. 1 shows the electrically conductive points (acupuncture points) on the human hands discovered by Dr. Voll. Western research found

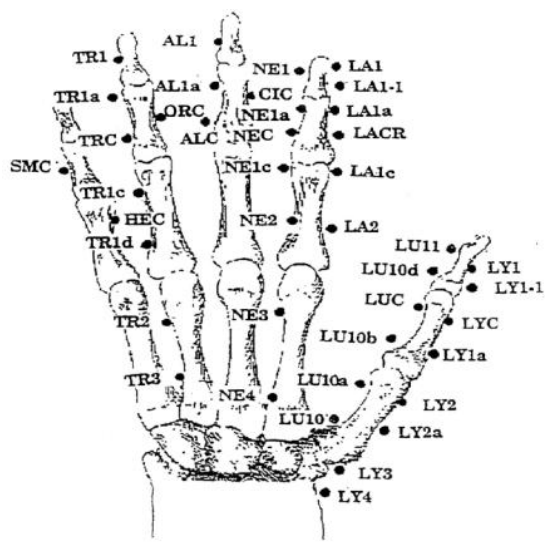

Fig. 1 Acupuncture points on the hand. that acupuncture points can be identified by low direct current resistance of the skin. In other words, acupuncture points are specific superficial anatomic locations. At these locations, the skin resistance is lower than that of the surrounding skin. Dr. Voll further discovered that the organ health condition can be determined by measuring the acupuncture impedance corresponding to the specific organs [1-2]. Besides, electric therapy studies showed that when therapy signals are repetitively directed into acupuncture points, the impedances of the acupuncture points can be restored, and the organs can be treated. Some patents disclose treatment methods or methods of monitoring the health of human bodies using acupuncture points [3-7].

In the past a bio-impedance measuring device and a metal probe are manually applied to measure the impedance of acupuncture point [1-7]. However, the accuracy of the measuring device is adversely affected by poor electrical contact between the probe and the skin, when one acupuncture point with typical area of $0.01 \mathrm{~cm}^{2}$ is measured, usually several measurements at different nearby locations are required to obtain stable and reliable skin resistance information. Some micro needles are made by polymer (SU-8 photo resist) [8-10], but they are used for transdermal drug delivery and not for electrical measurements. Moreover, some micro-array probes are made by silicon and metals [11-13] to solve the skin contact problem, the silicon micro needles are with flexibility by increasing the slenderness ratio and placing a polyimide on the back side, but they are still brittle, fragile and unable to dispose according to human body profile in a large area manner, thus the contact resistance between probe and skin may still be increased. Besides, those probes cannot piece into the corium layer and the acupuncture points usually lie under the thick cuticle, so the measured impedance is often higher than its true value, causing erroneous measuring results. Thus it is not only very time-consuming to make stable and reliable skin contact, but a trouble thing to measure the acupuncture impedance one by one. Although there are some other methods for health care [14-22], but they are not based on acupuncture impedance measurement. Due to the disadvantages of the previous impedance measuring devices, this paper proposes a new web-based health monitor device with multiple RFID tags

\footnotetext{
* This work is partially supported by National Science Council Taiwan, R.O.C. with contract NSC-101-2221-E-216-019- to Jium-Ming Lin.
} 
[23-28] as in Fig. 2, which can simultaneously measure the impedances of human acupuncture points. Each RFID tag comprises a flexible substrate, two sets of sharpened nonfragile micro-array probe, a RFID antenna, and a RFID IC chip embedded with a current driver and a measurement device. The micro-array probes are made of stainless steel covered with bio-compatible TiN, and they can pierce into the subject's corium layer by $200-300 \mu \mathrm{m}$ without penetrating the dermis and causing the infection problem. The tag is a flexible plastic substrate, e, g. PT, PET and PI, so the probes are easier to deploy and conform to the body skin profile. Besides, the measurement device can not only amplifier the bio-electrical signals to improve the $\mathrm{S} / \mathrm{N}$ ratio and impedance matching problems, but measure the acupuncture impedance [29-31]. Thus we can simultaneously deploy several RFID tags on the human's acupuncture points, and then remotely monitor and analyze the human health condition, and perform health care on the web. Thus the proposed system is very useful. Besides, several wearable fixtures on the body are also proposed.

The other parts of this paper are as follows: The second one concerns the operation principle. The next one relates the system integration test and discussion. The last part is the conclusion.

\section{Operation Principle}

The block diagram of the proposed web-based human health monitor with a RFID reader of monitor station and multiple RFID tags is shown in Fig. 2. As shown in Fig. 3 each RFID tag comprises a flexible substrate, two sets of sharpened non-fragile micro-array probes, an RFID antenna and a RFID IC chip embedded with a current driver and a measurement device. The micro-array probes are made of stainless steel covered with bio-compatible TiN. The tag is a flexible plastic substrate, e, g. PT, PET and PI, so the probes are easier to deploy, conform and touch the acupuncture points on the skin. As in Fig. 3, the RFID antenna is fabricated with PCB (Printed Circuit Board) technology on the substrate surface and configured to receive/transmit RF signals. The RFID IC chip on the right side is also bonded on the flexible substrate and coupled to the antenna.

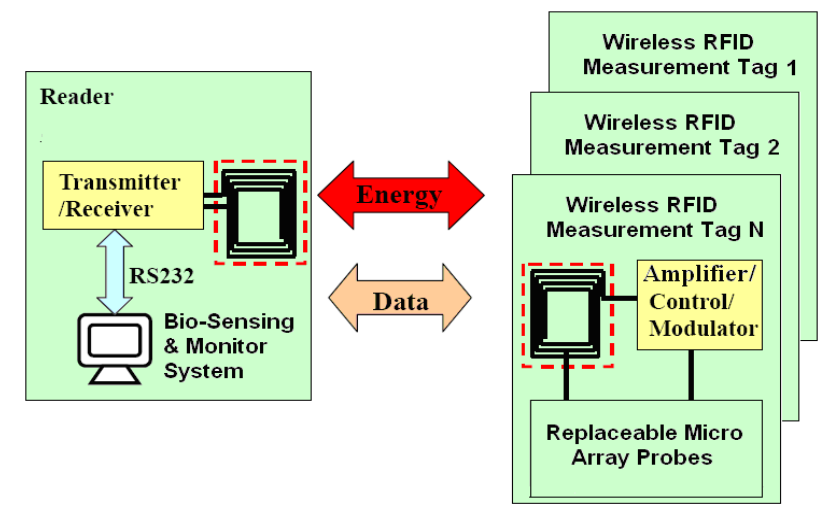

Fig. 2 Block diagram of web-based health monitor.

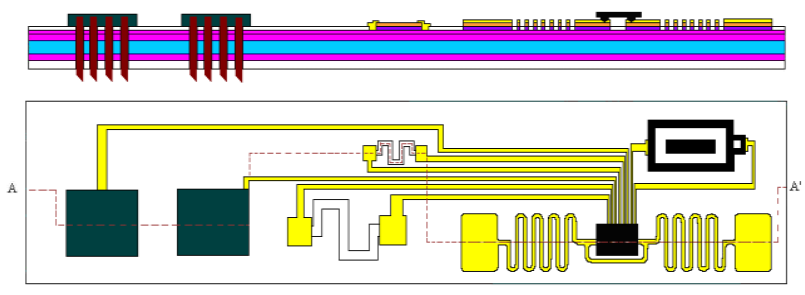

Fig. 3 Proposed active RFID tag with micro-array probes.

The RFID chip can demodulate the received RF signals from the web monitor station and measure the bio-potential of the acupuncture across the micro-array probes. Moreover, the probes on the left side protrude from the substrate are electrically-coupled to the measurement device embedded in RFID IC chip, the measurement device as shown in Fig. 4 can receive, amplify, and send the digitized bio-potential signals to RFID IC chip for encoding, and finally transmitted by the antenna to web monitor station (RFID reader) for monitoring and analysis. As shown in Fig. 4 the measurement device has a CMOS amplifier used for impedance matching. Since both the magnitudes of CMOS amplifier input impedance and bandwidth of are very large, thus not only S/N ratio but the impedance matching problem can be improved. Thus we can simultaneously deploy several RFID tags on the acupuncture points as shown in Figs. 5-7 for several wearable fixtures, and then monitor and analysis the acupuncture impedances and we can know the health condition on the web.

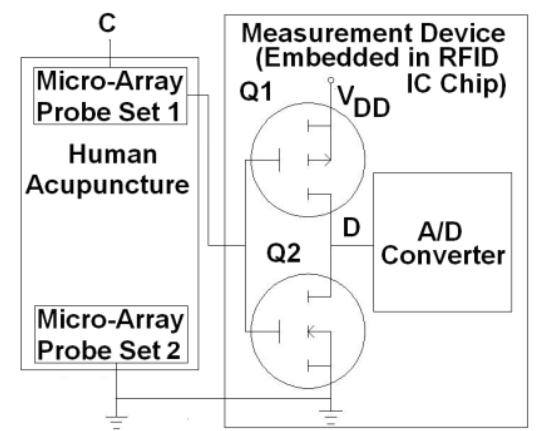

Fig. 4 System integration to send the bio-potential signals to RFID IC chip.

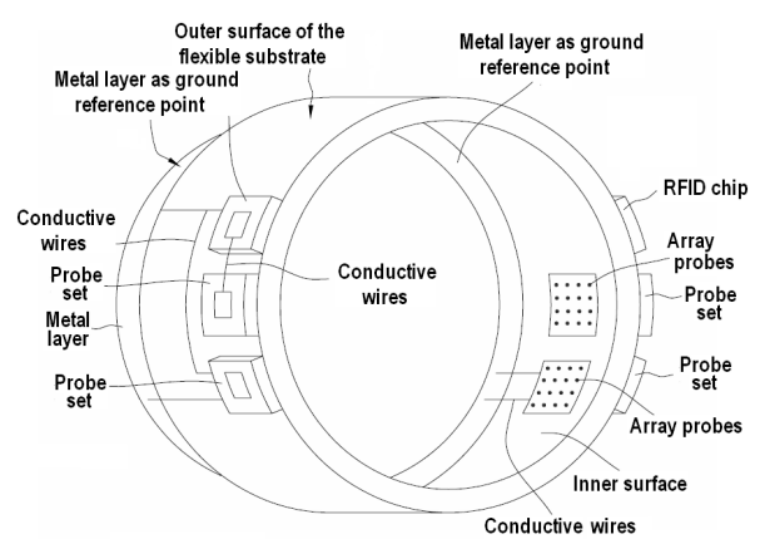

Fig. 5 Proposed bio-potential measurement device wrapped over a finger. 


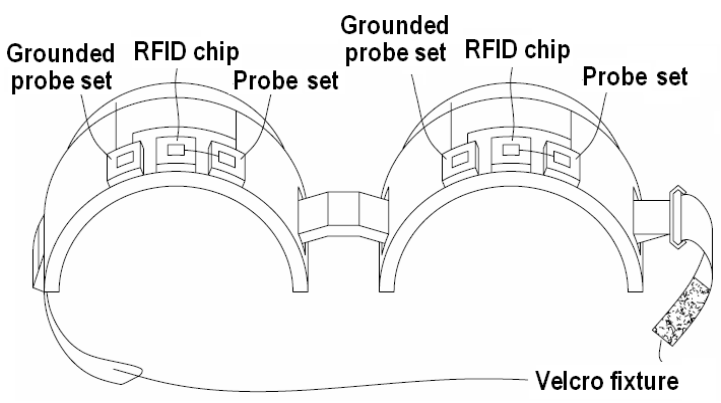

Fig. 6 Proposed bio-potential measurement device wrapped over a finger with a Velcro fixture.

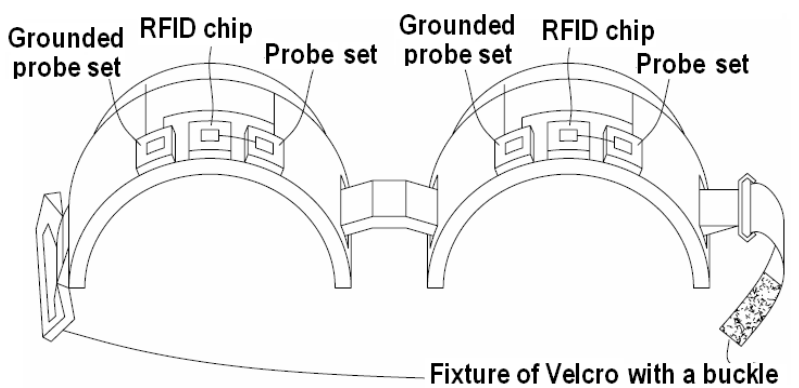

Fig. 7 Proposed bio-potential measurement device wrapped over a finger with a buckle on the Velcro fixture.

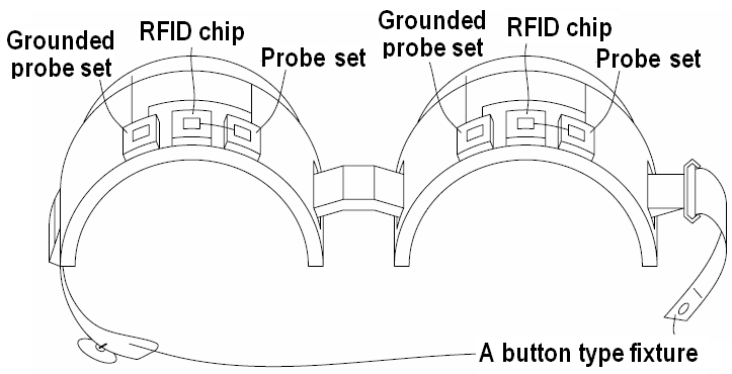

Fig. 8 Proposed bio-potential measurement device wrapped over a finger with a button type fixture.

\section{System Integration Test and Discussion}

As shown in Fig. 9 one set of the micro-probes is affixed over a man's acupuncture H5 (tōnglǐ) on the back of a wrist, and the other one is connected to the copper cylinder ground held by a hand. The RFID reader is placed at $15 \mathrm{~m}$ away. The bio-potentials for a man with and without staying up late for all night are obtained as shown in Fig. $10^{(29-31)}$. Noted that the bio-potential with staying up late for all night is much larger than the other one, we have repeated the test of the same person for several times, the results are almost in consistence with each other. Thus the bio-potentials obtained by the proposed device and system can be applied for the diagnosis, remote health care and monitoring of body organs. The other applications of the proposed apparatus are put multiple RFID tags on a glove wrapped over a finger, fingers and wrist as shown in Fig. 11, or fastened around the forehead as in Fig. 12, or on a sock wrapped around the leg and toe as in Fig. 13.

\section{Conclusions}

A RFID-based wireless bio-potential monitoring and health care system is proposed in this paper. The measurement device includes a flexible substrate configured to be fastened around a body portion, two probe sets attached to the flexible substrate, a RFID chip, and a wireless device. One probe set includes micro-array probes having tip portions configured to pierce the skin adjacent to an acupuncture point. Another probe set is used to contact the reference skin as an electrical ground. The measurement device is disposed on the flexible substrate and electrically coupled to the two probe sets to measure the bio-potentials between the acupuncture points and the reference ground point. Besides, the bio-potentials can be amplified and converted to a digital signal. The wireless device, coupled to the measurement device, is configured to transmit the acupuncture code and bio-potential information to a remote monitor station for the diagnosis and health care.

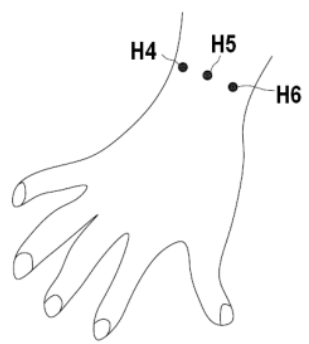

Fig. 9 Proposed bio-potential measurement device with a button type fixture.

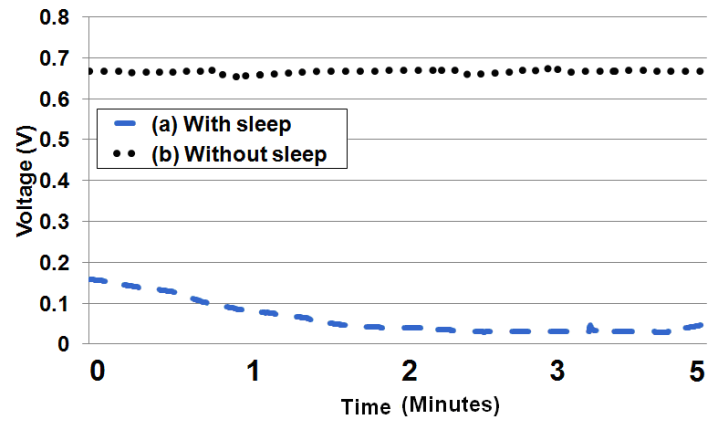

Fig. 10 Bio-potentials of a man (a) with and (b) without staying up late for all night.

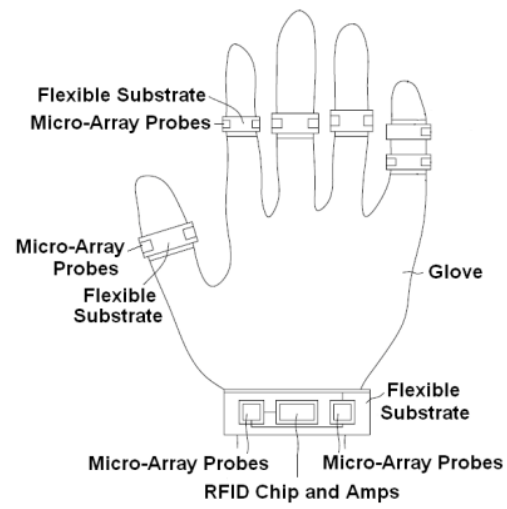

Fig. 11 The application by putting multiple tags on fingers and wrist. 


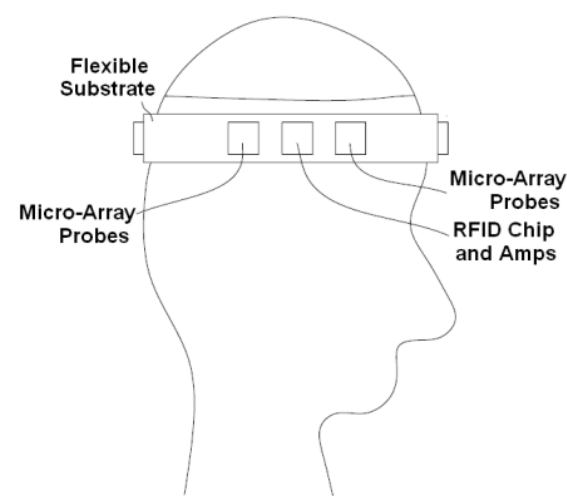

Fig. 12. The application by putting multiple RFID tags on the forehead.

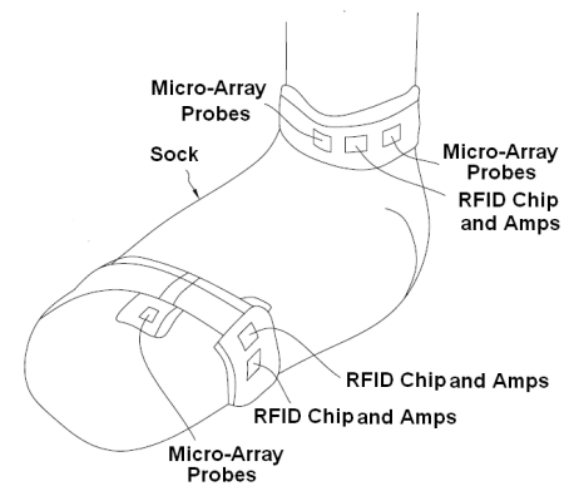

Fig. 13. The application by putting multiple RFID tags on the leg.

\section{Acknowledgment}

This work was supported by National Science Council Taiwan, R.O.C. with contract NSC-101-2221-E-216-019-.

\section{References}

[1] H. M. Johng, J. H. Cho, H. S. Shin, K. S. Soh, T. H. Koo, and S. Y. Choi, "Frequency dependence of impedances at the acupuncture point Quze (PC3)," IEEE Engineering in Medicine and Biology, vol. 21, no. 2, pp. $33-36,2002$.

[2] A. Ahn, A. P. Colber, B. J. Anderson, O. G. Martinsen, R. Hammerschlag, and S. Cina, "Electrical properties of acupuncture points and meridians: A systematic review," Bioelectromagnetics, vol. 29, no. 4 pp. 245-256, 2008

[3] L. E. Bertolucci, "Nausea control device," U.S. Patent 4,981,146, Jan 1 1991.

[4] T. L. Grey and L. E. Bertolucci, "Electrotherapy device," U.S. Patent 5,397,338, Mar 141995.

[5] B. Brewitt, "Methods for treating disorders by administering radio frequency signals corresponding to growth factors," U.S. Patent 5,626,617, May 61997.

[6] D. M. Giuntoli, G. J. Gruzdowich, and T. L. Grey, "Electro-acupuncture device with D-shaped stimulation electrodes," U.S. Patent No. 6,735,480, May 112004.

[7] O. Vahram, A. Valeri, M. Maksim, F. Gagik, and H. Minas, "Methods and devices for noninvasively measuring quantitative information of substances in living organisms," US Patent 2005/0197555, Sep 82005.

[8] X. Wang, B. Ma, Z. Gan, and H. Zhang, "Drop Test and Simulation of Portable Electronic Devices," 6th International Conference on Electronic Packaging Technology, pp. 1-4, 2005.

[9] B. Chen, J. Wei, E. Tay, Y. T. Wong, and C. Iliescu, "Silicon microneedle array with biodegradable tips for transdermal drug delivery," Microsyst. Technol., vol. 14, no. 7, pp. 1015-1019, 2008.
[10] J. H. Park, M. G. Allen, and M. R. Prausnitz, "Biodegradable polymer microneedles: fabrication, mechanics and transdermal drug delivery," Journal of Controlled Release, vol. 104, no. 1, pp. 51-66, 2005.

[11] L. Lin and A. Pisano, "Silicon-processed microneedles," Microelectromechanical Systems, vol. 8, no. 1, pp. 78-84, 1999.

[12] J. D. Zahn, N, H. Talbot, D. Liepmann, and A. P. Pisano, "Microfabricated polysilicon microneedles for minimally invasive biomedical devices," Biomedical Microdevices, vol. 2, no. 4, pp. $295-$ 303, 2000.

[13] M. Cormier, B. Johnson, and M. K. Ameri, "Fabrication and characterization of laser micromachined hollow microneedles," Transducers, Solid-State Sensors, Actuators and Microsystems, vol. 2, no. 2, pp. 1435-1438, 2003.

[14] C. C. Lin, M. J. Chiu, C. C. Hsiao, R. G. Lee, and Y. S. Tsai, "Wireless health care service system for elderly with dementia," IEEE Trans. Inf. Technol. Biomed., vol. 10, no. 4, pp. 696-704, 2006.

[15] A. Milenkovic, C. Otto, and E. Jovanov, "Wireless sensor networks for personal health monitoring: Issues and an implementation," Computer Communications, vol. 29, no. 13-14, pp. 2521-2523, 2006.

[16] E. Jovanov, A. Lords, D. Raskovic, P. Cox, R. Adhami, and F. Andrasik, "Stress monitoring using a distributed wireless intelligent sensor system," IEEE Engineering in Medicine and Biology Magazine vol. 22, no. 3, pp. 45-49, 2003.

[17] J. P. Lynch and K. J. Loh, "A Summary Review of Wireless Sensors and Sensor," The Shock and Vibration Digest, vol. 38, vo. 2, pp. 91-128, March 2006.

[18] E. Sardini and M. Serpelloni, "Instrumented wearable belt for wireless health monitoring," Procedia Engineering, vol. 5, pp. 580-583, 2010.

[19] G. López, V. Custodio, and J. I. Moreno, "LOBIN: E-textile and wireless-sensor-network-based platform for healthcare monitoring in future hospital environments," IEEE Transactions on Information Technology in Biomedicine, vol. 14, no. 6, pp. 1446-1458, 2010.

[20] H. Alemdar and C. Ersoy, "Wireless sensor networks for healthcare: A survey," Computer Networks, vol. 54, no. 15, pp. 2688-2710, 2010.

[21] Y. Xiao, D. Takahashi, J. Liu, H. Deng, and J. Zhang, "Wireless telemedicine and m-health: technologies, applications and research issues," International Journal of Sensor Networks, vol. 10, no. 4, pp. 202-236, 2011

[22] H. Huo, Y. Xu, H. Zhang, Y. H. Chuang, and T. C. Wu, "Wirelesssensor-networks-based healthcare system: a survey on the view of communication paradigms," International Journal of Ad Hoc and Ubiquitous Computing, vol. 8, no. 3, pp. 135-154, 2011.

[23] V. Chawla and D. S. Ha, "An overview of passive RFID," IEEE Appl. Practice, vol. 45, no. 9, pp. 11-17, 2007.

[24] W. Yao, C. H. Chu, and Zang Li, "Leveraging complex event processing for smart hospitals using RFID," Journal of Medical Systems, vol. 34, no. 3, pp. 799-810, 2011.

[25] M. Safkhani, N. Bagheri, M. Naderi, "On the designing of a tamper resistant prescription RFID access control system," Journal of Medical Systems, vol. 36, no. 6, pp. 3995-4004, 2012.

[26] E. W. T. Ngai, K. K. L. Moon, F. J. Riggins, and C. Y. Yi, "RFID research: An academic literature review (1995-2005) and future research directions," International Journal of Production Economics, vol. 112, no. 2, pp. 510-520, 2008.

[27] B. S. Ashar, and A. Ferriter, "Radiofrequency Identification Technology in Health CareBenefits and Potential Risks," The Journal of the American Medical Association, vol. 298, no.19, pp. 2305-2307, 2007.

[28] H. Alemdar and C. Ersoy, "Wireless sensor networks for healthcare: A survey," Computer Networks, Comput. Netw. vol. 54, no. 15, pp. 26882710, 2010.

[29] Y. Yamamoto, "Measurement and analysis of skin electrical impedance," Acta dermato-venereologica, Supplementum, vol. 185, pp. 34-38, 1994.

[30] K. Kontturi and L. Murtomäki, "Impedance Spectroscopy in Human Skin. A Refined Model," Pharmaceutical Research, vol. 11, no. 9, pp. 1355-1357, 1994

[31] K. G. Chen, "Electrical properties of meridians: with an overview of the electro-dermal screening test," IEEE Eng. Med./Biology Magazine, vol. 58, no. 3, pp. 58-63, 1996. 